The proof of the construction where $(\theta+\phi)$ is less than $180^{\circ}$ is illustrated in Fig. 3, where $A, B$ and $C$ are, as before, the observed marks and $P$ is the required position. AP is produced to cut the circle through $B C P$ in $E$ and $C P$ is produced to cut the circle through ABP in D.

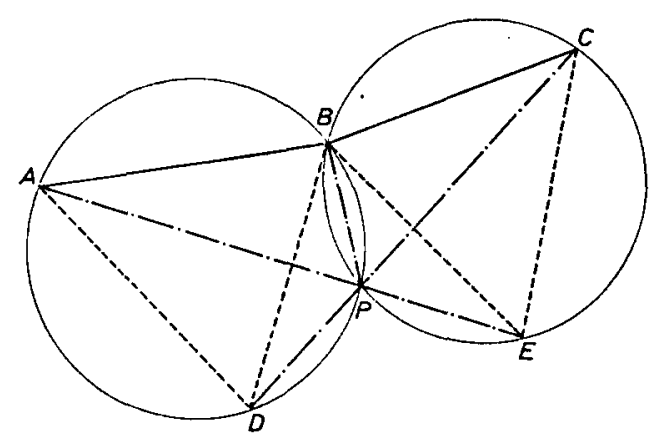

FIG. 3

Now $\Omega=\angle A B D=\angle A P D$ (since they are both subtended by the chord AD; Euclid III.21):

$$
\begin{aligned}
\angle \mathrm{APD} & =180^{\circ}-(\angle \mathrm{APB}+\angle \mathrm{BPC}) \\
\therefore \Omega & =180^{\circ}-(\theta+\phi) .
\end{aligned}
$$

Similarly $\Omega=\angle \mathrm{CBE}=\angle \mathrm{CPE}=180^{\circ}-(\theta+\phi)$.

In the cyclic quadrilaterals $\mathrm{ABPD}$ and $\mathrm{CBPE}$ :

$$
\begin{aligned}
& \angle \mathrm{BAD}=\angle \mathrm{BPC}=\phi \\
& \angle \mathrm{BCE}=\angle \mathrm{BPA}=\theta(\text { Euclid III.22). }
\end{aligned}
$$

A similar proof is applicable to the case where $(\theta+\phi)$ exceeds $180^{\circ}$.

Although in practice this method is no quicker to plot than other recognized methods, it does have the advantage of not requiring compasses. The construction is easily performed with a parallel ruler from the compass rose on the chart, but a circular or Douglas protractor is much more convenient.

The author wishes to thank Inst. Lt. Rickards, R.N. for reading the original paper and suggesting a clearer presentation.

\title{
The Calculation of Position Line Data with a Computer Calculator
}

\section{Henry L. Podmore}

As a result of the recent marketing of small pocket computers I have found much interest among navigators who would like to use them for working out 
celestial sights. One computer calculator (the Hewlett-Packard HP-35). in particular appears to be of considerable interest due to. its ability to compute trigonometric functions with algebraic logic as well as to store and recall derived intermediate values. Not having been able to find any published work on the use of this type of equipment for the rapid and precise working of sights I have given some thought to the matter and as a result believe the following notes may well be of interest to others.

The usual method of calculating zenith distance using the haversine formula overcomes the obvious difficulties of handling negative functions, but this computer calculator can handle negative values of sines and cosines without difficulty; moreover multiplication can be carried out without the need for logarithms. Hence the computer facilitates the use of the basic spherical cosine formula 1 which when applied to the PZX triangle may be expressed as :2

$$
\operatorname{Cos} \mathrm{P}=\frac{\operatorname{Cos} \mathrm{ZX}-(\operatorname{Cos} \mathrm{PZ})(\operatorname{Cos} \mathrm{PX})}{(\operatorname{Sin} \mathrm{PZ})(\operatorname{Sin} \mathrm{PX})}
$$

where $\mathrm{P}=$ Local Hour Angle

$\mathrm{ZX}=$ Zenith distance

$\mathrm{PZ}=$ Co-lat $\left(=90^{\circ}-\right.$ Lat $)$

$\mathrm{PX}=$ Polar distance $=90-\mathrm{Dec}$, where Lat and Dec are of the same name or $90+$ Dec (Lat and Dec Diff. Name)

Since $\operatorname{Sin} x=\operatorname{Cos}(90-x)$ we can substitute and transpose as follows :

$\operatorname{Cos}$ LHA $=\frac{\operatorname{Cos} Z X-(\operatorname{Sin} \text { Lat })(\operatorname{Sin} \text { Dec })}{\text { (Cos Lat) (Cos Dec) }}($ Lat and Dec Same Name)

$$
\text { or (Cos LHA) (Cos Lat) (Cos Dec) }=\operatorname{Cos} \mathrm{ZX}-(\operatorname{Sin} \text { Lat) (Sin Dec) }
$$

from which $\operatorname{Cos} \mathrm{ZX}=(\operatorname{Cos} \mathrm{LHA})$ (Cos Lat) (Cos Dec) + (Sin Lat) (Sin Dec). Where Lat and Dec have Different Names:

$$
\begin{aligned}
& \operatorname{Cos} \mathrm{ZX}=(\operatorname{Cos} \mathrm{LHA})(\text { Cos Lat) }(\operatorname{Sin} 90+\mathrm{Dec})+(\text { Sin Lat) }(\operatorname{Cos} 90+\mathrm{Dec}) \\
& \text { or } \operatorname{Cos} Z X=(\operatorname{Cos} L H A) \text { (Cos Lat) }(\operatorname{Cos} \text { Dec })+\text { (Sin Lat) }(-\operatorname{Sin} \text { Dec) } \\
& \operatorname{Cos} \mathrm{ZX}=(\operatorname{Cos} \mathrm{LHA}) \text { (Cos Lat) (Cos Dec) - (Sin Lat) (Sin Dec) }
\end{aligned}
$$

It will be seen that the formulae are the same for both conditions, except that when Lat and Dec have Different Names the arithmetic sign in the formula is negative. The formula in this form is eminently suitable for use on the computer, providing one first converts (on the computer) the various values expressed as degrees and minutes into degrees and fractions of a degree, to (say) 3 decimal places.

Let us consider the calculation of the zenith distance where :

$$
\begin{aligned}
\text { LHA } & =349.067^{\circ} \\
\text { D.R. Lat } & =53.167^{\circ} \mathrm{N} \\
\text { Dec } & =15.005^{\circ} \mathrm{N}
\end{aligned}
$$

Substituting in the above formula (Lat and Dec Same Name) we get:

$\operatorname{Cos} Z X=(\operatorname{Cos} 349.067)(\operatorname{Cos} 53.167)(\operatorname{Cos} 15.005)+\operatorname{Sin}(53.167)(\operatorname{Sin} 15.005)$

The necessary sequence of operations on the computer is shown below. The 
digital display (column 2) facilitates the entry of correct figures and accurate operation.

\begin{tabular}{|c|c|c|c|}
\hline & Press & See & Comments \\
\hline 1) & $\begin{array}{l}349 \cdot 067 \\
\operatorname{Cos}\end{array}$ & $\begin{array}{l}349 \cdot 067 \\
0.9818496387\end{array}$ & \\
\hline 3) & $53 \cdot 167$ & $53 \cdot 167$ & \\
\hline 4) & $\operatorname{Cos}$ & 0.5994846873 & Gives nroduct of values in frot two hizclete \\
\hline 5) & $\begin{array}{l}x \\
15.005\end{array}$ & $\begin{array}{l}0.5886038236 \\
15.005\end{array}$ & Gives product of values in hrst two brackets \\
\hline 7) & $\operatorname{Cos}$ & 0.9659032362 & \\
\hline 8) & $x$ & $0.56853433^{81}$ & Gives product of values in first three brackets \\
\hline 9) & STO & $0.56853433^{81}$ & $\begin{array}{l}\text { Storage of this result permits processing of last two } \\
\text { brackets }\end{array}$ \\
\hline 10) & $53 \cdot 167$ & $53 \cdot 167$ & \\
\hline II) & & 0.8003862253 & \\
\hline $\begin{array}{l}\text { I2) } \\
\text { I3) }\end{array}$ & $\begin{array}{l}15.005 \\
\text { Sin }\end{array}$ & $\begin{array}{l}15.005 \\
0.258903337\end{array}$ & \\
\hline 14) & $x$ & 0.2072226646 & Gives product of values in last two brackets \\
\hline 15) & RCL & $0.56853433^{81}$ & Recalls value in step 9 \\
\hline 16) & + & 0.7757570027 & Gives value of $\operatorname{Cos} \mathrm{ZX}$ \\
\hline $\begin{array}{l}\text { 17) } \\
\text { 18) }\end{array}$ & $\operatorname{arc}$ & 0.7757570027 & \\
\hline 19) & $\cos$ & $39 \cdot 12628446$ & Gives angle in degrees $(L X)$ having $\cos$ as in step 16 \\
\hline 20) & - & 0.12628446 & This leaves decimal degrees to be converted to mins \\
\hline 2I) & 60 & 60 & \\
\hline 22) & $x$ & $7 \cdot 5770676$ & Gives decimal degrees as minutes \\
\hline
\end{tabular}

Hence Zenith distance $=39^{\circ} 7 \cdot 6^{\circ}$

The standard program can be summarized thus:
r) LHA
2) $\operatorname{Cos}$
3) D.R. Lat
4) $\operatorname{Cos}$
5) $x$
6) $\mathrm{Dec}$
7) $\operatorname{Cos}$
8) $x$
9) $\mathrm{STO}$
Io) D.R. Lat
I I) $\mathrm{Sin}$
12) Dec
13) $\mathrm{Sin}$
14) $x$
15) $\mathrm{RCL}$
16) $\left\{\begin{array}{l}+ \text { (If Lat and Dec Same Name) } \\ x^{\infty} y \text { followed by - (If Lat and Dec Different Name) }\end{array}\right.$
I7) arc
18) $\mathrm{Cos}$
19) Enter i 8 as whole number \& note result (Z.D. in degrees) 
20) -

21) 60

22) $\times$ Note minutes to first decimal place

Steps 19 and 22 combine to give the ZD in degrees and minutes.

Recently Hewlett-Packard have introduced a new instrument, the HP-65, which enables the above program to be recorded on magnetic tape and then subsequently used to calculate automatically the ZD for any given values of LHA, D.R. Lat and Dec. This particular computer handles degrees, minutes and seconds directly - which can be an advantage.

The calculation of LHA is, of course, carried out in the usual manner. The intercept is also found in the normal way by comparing the calculated zenith distance with the observed zenith distance. The azimuth can easily be found from $\mathrm{ABC}^{3}$ or similar tables.

The time taken to plot a position line using this method is appreciably less than can be achieved by the use of sight reduction tables. Moreover, while it is agreed that reduction tables provide adequate accuracy, the possibility of making an error, particularly in interpolation, is much more likely than when using a computer calculator.

The main advantage, however, of the proposed method results from the fact that the position line can be plotted from the D.R. position-there is no need to work out a chosen position. Hence if a number of simultaneous sights are used they can all be plotted from the same point, i.e. the D.R. or Estimated Position of the ship. It follows that if the Estimated Position is reasonably accurate and the plot is made from this position, then the intercept will be short and the accuracy of the plot correspondingly high. 4

\title{
REFEREN CES
}

1 Brown, H. H. Nicholl's Concise Guide, volume II. Glasgow.

2 Cotter, C. H. The Complete Nautical Astronomer. London.

3 Norie, J. W. Norie's Nautical Tables by F. N. Hopkins, London.

4 Admiralty Manual of Navigation, volume II. London.

\section{Air Navigation with a Pocket Electronic Calculator}

\author{
J. D. Proctor
}

Some navigators may prefer other methods and other tools, but the Hewlett Packard $\mathrm{HP}-45$ has some fascinating abilities. It might be called the poor man's INS computer, though it does not of course find position. Its only disadvantages are the need for a hood in sunlight and the need for concentration and freedom from distraction. It can be used on the knee for no table is required, and it replaces conversion tables and several other mathematical tables. To a large extent it can replace plotting charts, flight planning charts, Dalton computers, protractors and dividers.

It simplifies ground speed/distance/time and fuel calculations especially, with its ability simply to convert hours, minutes and seconds or degrees, minutes and seconds to and from decimals. It calculates in the decimal mode. 